\title{
Non-animal-derived monoclonal antibodies
} are not ready to substitute current hybridoma
technology

To the Editor - We write on behalf of the COSCE (Confederation of Spanish Scientific Societies) Transparency Agreement on Animal Research, supported by the EARA (European Animal Research Association). In May 2020, the European Commission's Joint Research Centre (EC-JRC) released a recommendation on the development of non-animal-derived antibodies, urging government authorities, funding agencies and publishers to endorse the use of these antibodies to improve scientific reproducibility ${ }^{1}$. These recommendations were based on the work done by the Scientific Advisory Committee (ESAC) of the European Union Reference Laboratory for alternatives to animal testing (EURL ECVAM). Recent correspondence to Nature $^{2}$ and Nature Methods ${ }^{3}$ claims that non-animal antibodies are ready to replace animal-derived ones in all known applications. In our view, however, both the EC-JRC document and the published correspondence contain distorted perceptions of the current possibilities for non-animal-derived antibodies. While we are all committed to replacing animal experimentation with alternative methods, these methods need further scientific validation to justify replacing the use of animals without affecting the desired outcome of the experiment.

In our opinion, there are still major concerns barring the substitution of hybridoma technology with animal-free methods, such as insufficient technological development, inconsistent efficiency depending on the application, and difficulty in implementation at a global scale.

There are no universal (phage, yeast, ribosome or mRNA) display technologies yet, and in many cases animal immunization is still needed. In addition, display technologies have important limitations when generating antibodies against native structures ${ }^{4}$ - for example, viruses. In vivo somatic hypermutation allows the development of monoclonal antibodies with much higher affinity and specificity than those generated by phage technology ${ }^{5}$. While efforts have been made to improve the quality of display-based antibodies, further development requiring substantial experimentation, time and resources is still needed. By contrast, hybridoma technology is well established and allows the isolation of native antibodies generated in the context of an immune response against a given antigen. During vaccine development, this method provides valuable information on how the immune system reacts to that antigen, including the immunoglobulin genes involved, secondary reordering, insertions and deletions, affinity maturation, the clonal relationships of different antibodies, isotypes ${ }^{6,7}$ etc. All this crucial information is lost when using display technology.

It is important to highlight that

hybridoma generation only requires animals during the immunization phase. The next steps - for example, cellular fusion, clonal selection and antibody production - are animal-free, and large quantities of monoclonal antibodies for therapeutic use are currently being produced by genetically modified cells at an industrial scale. Thus, the antibody production field is mostly animal-free already, and protocols for immunization have been improved to maximize animal welfare. This includes the use of better adjuvants with fewer secondary effects, reduced number of immunizations, and improved routes of immunization.

Display technologies can have advantages in some fields (for example, toxicology, antivenom research) but do not work equally well for other applications, such as therapeutic antibody development, and for analytical purposes ${ }^{8}$. The two techniques are complementary ${ }^{9}$, and the transition toward non-animal-derived antibodies needs to take into account these differences in applications ${ }^{8,10}$.

Finally, the implementation of new technologies in a laboratory requires staff training and an adaptation period. There are as yet very few laboratories in the world with access to display technologies or other animal-free systems. ESAC members are aware of these limitations, as they state in their recommendation ${ }^{1}$, implicitly acknowledging the enormous difficulty in implementing display-based technology. To improve affinity and specificity, antibody-producing companies have the resources to use large, diverse libraries, high-throughput selection methods to pick ideal binders, and careful engineering strategies to further improve them if necessary. By contrast, the scenario is very different for small research laboratories without experience in display techniques, or without powerful libraries and selection platforms.

Unfortunately, display technologies are still not a real alternative to hybridoma technology for all applications, and therefore we believe that the endorsement promoted by the EC-JRC is not scientifically justified. The ideal scenario is to have both and to choose the best method in every case according to the particular application. Any change should be supported by scientific evidence and monitored by regulatory bodies, independent of third-party interests. Use of animal-free methods should be limited to those fields where it has been demonstrated to be a real alternative to traditional technology, while developing a roadmap that allows a successful transition to these new technologies and thus really benefiting society.

\section{África González-Fernández (DD) 1⿴, Francisco J. Bermúdez Silva (D), Marcos López-Hoyos (D) ${ }^{3}$, César Cobaleda (D), Lluís Montoliu (D) 5 , Margarita Del Val (D)6 and Kirk Leech ${ }^{7}$ ${ }^{1}$ CINBIO, Centro de Investigaciones Biomédicas, Universidade de Vigo, Vigo, Spain. ${ }^{2} U G C$} Endocrinología y Nutrición, Instituto de Investigación Biomédica de Málaga-IBIMA, Hospital Regional

Universitario de Málaga, Málaga, Spain. ${ }^{3}$ Immunology Service, Hospital Universitario Marqués de Valdecilla-IDIVAL, Santander, Spain. ${ }^{4}$ Immune System Development and Function Unit. Department of Cell Biology and Immunology, Centro de Biología Molecular Severo Ochoa (Consejo Superior de Investigaciones Científicas Universidad Autónoma de Madrid) (CBMSO-CSIC/ UAM), Madrid, Spain. ${ }^{5}$ Animal Models by Genetic Manipulation Unit, National Centre for Biotechnology (Consejo Superior de Investigaciones Cientificas) (CNB-CSIC) and Biomedical Research Networking Center on Rare Diseases (Instituto de Salud Carlos III) (CIBERER-ISCIII), Madrid, Spain. ${ }^{6}$ Viral Immunology, Centro de Biología Molecular Severo Ochoa (Consejo Superior de Investigaciones Científicas - Universidad Autónoma de Madrid) (CBMSO, CSIC-UAM), Madrid, Spain. ${ }^{7}$ European Animal Research Association (EARA), London, UK.

$\bigotimes_{e \text {-mail: africa@uvigo.es }}$ 
Published online: 5 October 2020

https://doi.org/10.1038/s41592-020-00977-5

References

1. Viegas Barroso, J., Halder, M. E. \& Whelan, M. EURL ECVAM recommendation on non-animal-derived antibodies, EUR 30185 EN (European Union, 2020); https://doi.org/10.2760/80554

2. Gray, A. C. et al. Nature 581, 262 (2020)

3. Gray, A. C. et al. Nat. Methods 17, 755-756 (2020).

4. Jones, M. L. et al. Sci. Rep. 6, 26240 (2016).
5. Sorouri, M., Fitzsimmons, S. P., Aydanian, A. G., Bennett, S. \& Shapiro, M. A. PLoS ONE 9, e106699 (2014).

6. Kurosawa, K. \& Ohta, K. Genes (Basel) 2, (48-58 (2011).

7. Di Noia, J. M. \& Neuberger, M. S. Annu. Rev. Biochem. 76, 1-22 (2007).

8. Zaroff, S. \& Tan, G. Biotechniques 67, 90-92 (2019).

9. Rossant, C. J. et al. MAbs 6, 1435-1438 (2014).

10. Ch'ng, A. C. W., Choong, Y. S. \& Lim, T. S. In Proof and Concepts in Rapid Diagnostic Tests and Technologies (ed. Saxena, S. K.) (InTech, 2016).

\section{Acknowledgements}

We thank the COSCE societies for their support (https://cosce.org/).

Author contributions

The authors contributed equally to this work.

Competing interests

A.G.-F. was cofounder of the spinoff company NanoImmunotech.

\section{The Most Advanced Patch Clamp System Available Today}

\section{Visit www.dpatch.com to learn more}

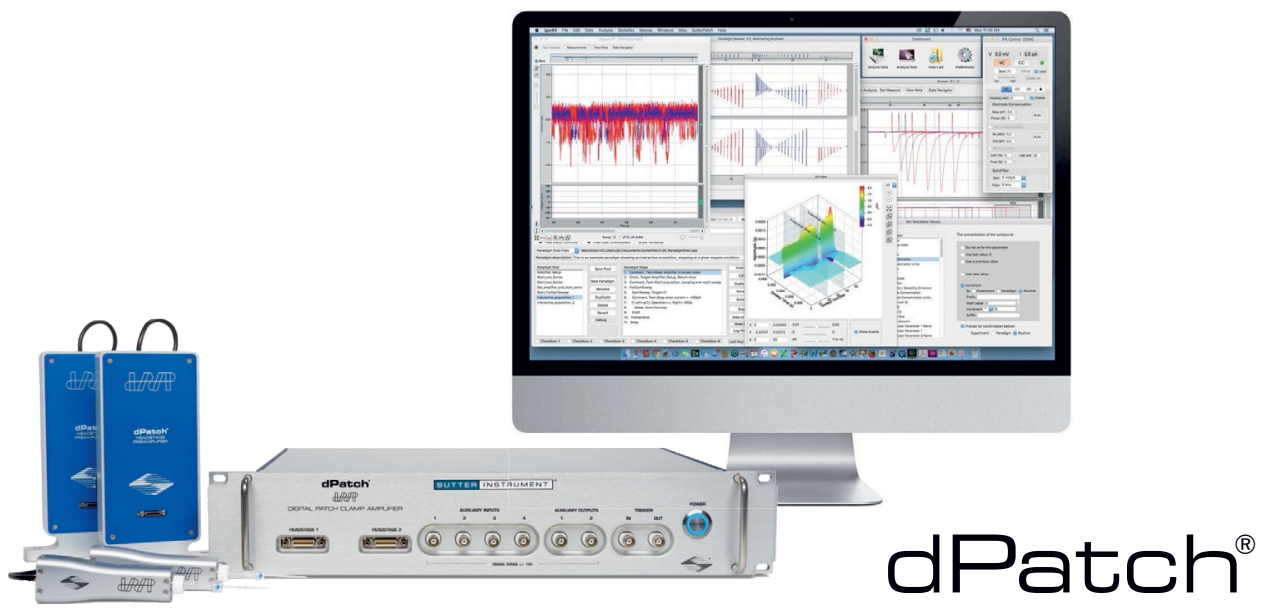

\section{SUTTER INSTRUMENT}

